\title{
ON APPROXIMATION OF COPULAS
}

\author{
TOMASZ KULPA
}

(Received 6 May 1997 and in revised form 22 October 1997)

\begin{abstract}
New sufficient conditions for strong approximation of copulas, generated by sequences of partitions of unity, are given. Results are applied to the checkerboard and Bernstein approximations.
\end{abstract}

Keywords and phrases. Copulas, Markov operators, doubly stochastic measures.

1991 Mathematics Subject Classification. 60B10, 47B65, 41A35, 28A35.

1. Introduction. A copula is a distribution function of a doubly stochastic measure $\mu$ on the unit square $[0,1]^{2}$, i.e., $C(x, y)=\mu([0, x] \times[0, y])$ for $x, y \in[0,1]$. Copulas are of interest because they link joint distributions to marginal distributions. Sklar showed in $[8,9]$ that, for any real-valued random variables $X_{1}$ and $X_{2}$ with joint distribution $F_{12}$, there is a copula $C$ such that

$$
F_{12}\left(x_{1}, x_{2}\right)=C\left(F_{1}\left(x_{1}\right), F_{2}\left(x_{2}\right)\right)
$$

where $F_{1}$ and $F_{2}$ denote the cumulative distribution functions of $X_{1}$ and $X_{2}$, respectively.

Copulas are Lipschitz functions and the set of copulas is a convex compact subset of the space of continuous functions with uniform norm. Therefore, a natural way of approximating copulas is approximation in the topology of uniform convergence. The copula captures information about the dependence structure of $X_{1}$ and $X_{2}$. It is surprising that any copula, even the copula which relates a pair of independent random variables, can be approximated arbitrarily closely in the uniform sense by copulas which correspond to the deterministic dependence between a pair of random variables. Li, Mikusiński, Sherwood, and Taylor, in their work [5, 6], proposed another type of convergence of copulas. Namely, since the set of copulas is isomorphic to the set of Markov operators on $L^{\infty}[0,1]$, a strong convergence of copulas is defined by the strong convergence of the corresponding Markov operators. This convergence does not lead to the paradox mentioned above.

In [5, 6], Li, Mikusiński, Sherwood, and Taylor discussed sequences of approximation copulas given by partitions of unity. The aim of this paper is to give sufficient conditions for these sequences to be convergent in the strong sense. The convergence in the strong operator topology of $L^{p}(p \geq 1)$ is also discussed.

The paper is organized as follows: Section 2 contains preliminary definitions and results. In Section 3, we formulate and prove theorems concerning the convergence of Markov operators. In Section 4, Corollary 4.5 gives sufficient conditions for the strong 
convergence of Markov operators related to partitions of unity. In Section 5, we give three examples of approximation: the checkerboard, Bernstein, and tent approximations. Similar results for the checkerboard and Bernstein approximations were proved in [6] by different methods.

2. Preliminaries. A copula is a function $C:[0,1]^{2} \rightarrow[0,1]$ satisfying the boundary conditions $C(x, 0)=C(0, y)=0, C(x, 1)=x, C(1, y)=y$, and the monotonicity condition

$$
C\left(x_{2}, y_{2}\right)-C\left(x_{2}, y_{1}\right)-C\left(x_{1}, y_{2}\right)+C\left(x_{1}, y_{1}\right) \geq 0
$$

for all $x_{1}, y_{1}, x_{2}, y_{2} \in[0,1]$ satisfying $x_{1} \leq x_{2}$ and $y_{1} \leq y_{2}$.

We say that $T: L^{\infty}[0,1] \rightarrow L^{\infty}[0,1]$ is a Markov operator if it satisfies the following three conditions

$$
\begin{aligned}
T(f) & =f \quad \text { if } f(x)=1 ; \\
T(f) & \geq 0 \quad \text { for } f \geq 0 ; \\
\int_{0}^{1} T f(t) d t & =\int_{0}^{1} f(t) d t \quad \text { for all } f \in L^{\infty} .
\end{aligned}
$$

A Markov operator is bounded and the norm of $T$ in $L^{\infty}$ is 1 . We remark that $T$ can be extended to $L^{p}(p \geq 1)$ and it is easy to verify (by (2.2) and (2.4)) that the norm of $T$ in $L^{1}$ is 1 . Therefore, by the Riesz-Thorin interpolation theorem, the norm of $T$ in $L^{p}$ is also 1 . The set of copulas is isomorphic to the set of Markov operators $T$ on $L^{\infty}[0,1]$ via the correspondence

$$
\begin{aligned}
& \left(T_{C} f\right)(x)=\frac{d}{d x} \int_{0}^{1} C_{, 2}(x, t) f(t) d t, \\
& C_{T}(x, y)=\int_{0}^{x}\left(T \chi_{[0, y]}\right)(s) d s,
\end{aligned}
$$

where $C_{, 2}=\partial C / \partial y$.

We say that $C_{n}$ converges to $C$ in the strong sense if $T_{C_{n}}$ converges to $T_{C}$ in the strong operator topology of $L^{1}$. This strong convergence has a probabilistic interpretation. Let $T$ be a Markov operator. $T$ corresponds to some doubly stochastic measure on $[0,1]^{2}$ so that we may regard $[0,1]^{2}$ as a probability space. Then

$$
T f(x)=E[f(Y): X=x] \text { a.e., }
$$

that is, $T f(x)$ is the mean value of $f(Y)$ given that $X=x$, where $f$ is a real-valued function on $[0,1]$ and $X, Y$ are random variables on $[0,1]^{2}$ defined by $X(u, v)=u$ and $Y(u, v)=v$. Therefore, strong convergence for copulas amounts to convergence of conditional expectations.

3. Convergence of Markov operators. We study the following situation. Let $k_{n}$ : $[0,1]^{2} \rightarrow R$ be a sequence of nonnegative measurable functions satisfying the following two conditions 


$$
\begin{array}{ll}
\int_{0}^{1} k_{n}(x, y) d x=1 & \text { for a.e. } y \in[0,1] \\
\int_{0}^{1} k_{n}(x, y) d y=1 & \text { for a.e. } x \in[0,1]
\end{array}
$$

It is easy to verify that the operators $P_{n}, P_{n}^{*}: L^{\infty}[0,1] \rightarrow L^{\infty}[0,1]$ defined by

$$
\begin{aligned}
& P_{n} f(x)=\int_{0}^{1} k_{n}(x, y) f(y) d y, \\
& P_{n}^{*} f(y)=\int_{0}^{1} k_{n}(x, y) f(x) d x
\end{aligned}
$$

are Markov operators. Now, we formulate two theorems.

THEOREM 3.1. Let $k_{n}:[0,1]^{2} \rightarrow R$ be a sequence of nonnegative measurable functions satisfying (3.1), (3.2), and

$$
\lim _{n \rightarrow \infty} \iint_{A(\varepsilon)} k_{n}(x, y) d x d y=1 \text { for every } \varepsilon>0,
$$

where $A(\varepsilon)=\left\{(x, y) \in[0,1]^{2}:|x-y|<\varepsilon\right\}$. Then $P_{n} \rightarrow I$ and $P_{n}^{*} \rightarrow I$ in the strong operator topology of $L^{1}$, where $I$ is the identity operator.

THEOREM 3.2. Let $k_{n}:[0,1]^{2} \rightarrow R$ be a sequence of nonnegative measurable functions satisfying (3.1), (3.2), and, for every $\varepsilon>0$,

$$
\begin{array}{ll}
\lim _{n \rightarrow \infty} \int_{A(\varepsilon, y)} k_{n}(x, y) d x=1 & \text { for a.e. } y \in[0,1], \\
\lim _{n \rightarrow \infty} \int_{A(\varepsilon, y)} k_{n}(x, y) d y=1 & \text { for a.e. } x \in[0,1],
\end{array}
$$

where $A(\varepsilon, z)=(z-\varepsilon, z+\varepsilon) \cap[0,1]$. Then $P_{n} \rightarrow I$ and $P_{n}^{*} \rightarrow I$ in the strong operator topology of $L^{p}, p \in[1, \infty)$, where I is the identity operator.

Observe that every sequence of functions satisfying assumptions (3.6) and (3.7) of Theorem 3.2 also satisfies condition (3.5) of Theorem 3.1. Usually, it is easier to verify condition (3.5).

Now, we prove Theorem 3.1 and 3.2. First, we recall the following lemma, given in [6, Lem. 3.1].

LEMMA 3.3. Let $P_{n}, n=1,2, \ldots$, and $P$ be Markov operators. Then the following three statements satisfy: (i) $\Rightarrow$ (ii) $\Leftrightarrow$ (iii).

(i) For $f(x)=\chi_{[0, \lambda]}(x)$ and for a.e. $\lambda \in[0,1]$,

$$
\lim _{n \rightarrow \infty} P_{n}(f)(x)=P(f)(x) \text { a.e. on }[0,1] \text {. }
$$

(ii) For $f(x)=x_{[0, \lambda]}(x)$, for a.e. $\lambda \in[0,1]$, and for every $p \in[1, \infty)$,

$$
\lim _{n \rightarrow \infty}\left\|P_{n}(f)-P(f)\right\|_{p}=0 .
$$

(iii) $P_{n} \rightarrow P$ in the strong operator topology of $L^{p}$ for every $p \in[1, \infty)$, i.e.,

$$
\lim _{n \rightarrow \infty}\left\|P_{n}(f)-P(f)\right\|_{p}=0 \quad \text { for every } f \in L^{p} .
$$


Proof OF Theorem 3.2. According to Lemma 3.3, we need only to show that for a.e. $\lambda \in[0,1]$

$$
\lim _{n \rightarrow \infty} P_{n}\left(\chi_{[0, \lambda]}\right)(x)=I\left(\chi_{[0, \lambda]}\right)(x)=\chi_{[0, \lambda]}(x) \text { a.e. }
$$

Applying Definition (3.3), we obtain

$$
\begin{aligned}
\lim _{n \rightarrow \infty} P_{n}\left(\chi_{[0, \lambda]}\right)(x) & =\lim _{n \rightarrow \infty} \int_{0}^{1} k_{n}(x, y) \chi_{[0, \lambda]}(y) d y \\
& =\lim _{n \rightarrow \infty} \int_{0}^{\lambda} k_{n}(x, y) d y .
\end{aligned}
$$

Let $x<\lambda$ and $\varepsilon=\lambda-x$. From (3.2) and (3.7), it follows

$$
1=\int_{0}^{1} k_{n}(x, y) d y \geq \int_{0}^{\lambda} k_{n}(x, y) d y \geq \int_{A(\varepsilon, x)} k_{n}(x, y) d y \longrightarrow 1 .
$$

For $x>\lambda$ and $\varepsilon=x-\lambda$, we have

$$
0 \leq \int_{0}^{\lambda} k_{n}(x, y) d y \leq \int_{[0,1] \backslash A(\varepsilon, x)} k_{n}(x, y) d y=1-\int_{A(\varepsilon, x)} k_{n}(x, y) d y \longrightarrow 0 .
$$

This shows that

$$
\lim _{n \rightarrow \infty} P_{n}\left(\chi_{[0, \lambda]}\right)(x)=\lim _{n \rightarrow \infty} \int_{0}^{\lambda} k_{n}(x, y) d y=\chi_{[0, \lambda]}(x) \text { for a.e. } x \in[0,1] .
$$

Thus, $P_{n} \rightarrow I$ in the strong operator topology of $L^{p}$. The proof that $P_{n}^{*} \rightarrow I$ is analogous.

Proof OF Theorem 3.1. We only show that $P_{n} \rightarrow I$ in the strong operator topology of $L^{1}$. The proof that $P_{n}^{*} \rightarrow I$ is analogous. Let $\varphi_{n, \varepsilon}(x)=\int_{A(\varepsilon, x)} k_{n}(x, y) d y$. First, we claim that $\varphi_{n, \varepsilon} \rightarrow \chi_{[0,1]}$ in $L^{1}$ norm. Since

$$
0 \leq \varphi_{n, \varepsilon}(x)=\int_{A(\varepsilon, x)} k_{n}(x, y) d y \leq \int_{0}^{1} k_{n}(x, y) d y=1 \quad \text { a.e., }
$$

we have

$$
\begin{aligned}
\left\|\varphi_{n, \varepsilon}-\chi_{[0,1]}\right\|_{1} & =\int_{0}^{1}\left|\varphi_{n, \varepsilon}(x)-\chi_{[0,1]}(x)\right| d x \\
& =1-\int_{0}^{1} \int_{A(\varepsilon, x)} k_{n}(x, y) d y d x \\
& =1-\iint_{A(\varepsilon, x)} k_{n}(x, y) d y d x \rightarrow 0 .
\end{aligned}
$$

This implies that, for $\lambda \in[0,1]$,

$$
\int_{0}^{\lambda}\left|\chi_{[0, \lambda]}(x)-\varphi_{n, \varepsilon}(x)\right| d x \rightarrow 0 .
$$

Now, we show that $P_{n} \rightarrow I$. Let $f(x)=\chi_{[0, \lambda]}(x)$, then 


$$
\begin{aligned}
\left\|P_{n}(f)-f\right\|_{1} & =\int_{0}^{1}\left|P_{n}\left(\chi_{[0, \lambda]}\right)(x)-\chi_{[0, \lambda]}(x)\right| d x \\
& =\int_{0}^{1}\left|\int_{0}^{1} k_{n}(x, y) \chi_{[0, \lambda]}(y) d y-\chi_{[0, \lambda]}(x)\right| d x \\
& =\int_{0}^{\lambda}\left(1-\int_{0}^{\lambda} k_{n}(x, y) d y\right) d x+\int_{\lambda}^{1} \int_{0}^{\lambda} k_{n}(x, y) d y d x \\
& =J_{n, 1}+J_{n, 2} .
\end{aligned}
$$

We claim that $J_{n, 1} \rightarrow 0$ and $J_{n, 2} \rightarrow 0$. For $\varepsilon \in(0, \lambda]$, we have

$$
\begin{aligned}
0 \leq J_{n, 1} & =\int_{0}^{\lambda}\left(1-\int_{0}^{\lambda} k_{n}(x, y) d y\right) d x \\
& =\lambda-\int_{0}^{\lambda} \int_{0}^{\lambda} k_{n}(x, y) d y d x \\
& \leq \lambda-\int_{0}^{\lambda-\varepsilon} \int_{0}^{\lambda} k_{n}(x, y) d y d x \\
& \leq \lambda-\int_{0}^{\lambda-\varepsilon} \int_{A(\varepsilon, x)} k_{n}(x, y) d y d x \\
& =\varepsilon+\int_{0}^{\lambda-\varepsilon}\left(\chi_{[0, \lambda-\varepsilon]}(x)-\varphi_{n, \varepsilon}(x)\right) d x \leq 2 \varepsilon
\end{aligned}
$$

for sufficiently large $n$ because of (3.18). Now, choose $\varepsilon \in(0,1-\lambda]$. Observe that

$$
\begin{aligned}
0 \leq J_{n, 2} & =\int_{\lambda}^{1} \int_{0}^{\lambda} k_{n}(x, y) d y d x \\
& =\int_{\lambda}^{\lambda+\varepsilon} \int_{0}^{\lambda} k_{n}(x, y) d y d x+\int_{\lambda+\varepsilon}^{1} \int_{0}^{\lambda} k_{n}(x, y) d y d x \\
& \leq \int_{\lambda}^{\lambda+\varepsilon} \int_{0}^{1} k_{n}(x, y) d y d x+\iint_{[0,1]^{2} \backslash A(\varepsilon)} k_{n}(x, y) d y d x \\
& =\varepsilon+1-\iint_{A(\varepsilon)} k_{n}(x, y) d y d x \leq 2 \varepsilon
\end{aligned}
$$

for sufficiently large $n$. The set of characteristic functions of intervals $[0, \lambda], \lambda \in[0,1]$, is a linearly dense subset of $L^{1}$. Since $P_{n}, n=1,2, \ldots$, are linear contractions, $P_{n}(f) \rightarrow f$ for every $f \in L^{1}([0,1])$. This completes the proof in view of Lemma 3.3.

4. Partition of unity operators. In some applications, we are interested in approximations of copulas by simple ones. One type of approximation is related to the partitions of unity. We recall the definition

DEFINITION 4.1. A collection of functions $\phi_{1}, \ldots, \phi_{n} \in L^{1}([0,1])$ is called a partition of unity if

$$
\begin{aligned}
\phi_{i} \geq 0 & \text { for } i=1, \ldots, n \\
\int_{0}^{1} \phi_{i}(x) d x=\frac{1}{n} & \text { for } i=1, \ldots, n \\
\sum_{i=1}^{n} \phi_{i}(x)=1 & \text { for every } x \in[0,1]
\end{aligned}
$$


This approximation of copulas using the sequence of partitions of unity is given in [5, Thm. 6].

Proposition 4.2. Let $\phi_{1}, \ldots, \phi_{n} \in L^{1}([0,1])$ be nonnegative functions. The following statements are equivalent

(i) $\phi_{1}, \ldots, \phi_{n}$ is a partition of unity.

(ii) For every copula $C$, the operator $T_{n}(C): L^{1} \rightarrow L^{1}$ defined by

$$
T_{n}(C)(f)(x)=n^{2} \sum_{i, j=1}^{n} \Delta_{i, j}(C)\left(\int_{0}^{1} \phi_{j}(y) f(y) d y\right) \phi_{i}(x)
$$

is a Markov operator, where

$$
\Delta_{i, j}(C)=C\left(\frac{i}{n}, \frac{j}{n}\right)-C\left(\frac{i-1}{n}, \frac{j}{n}\right)-C\left(\frac{i}{n}, \frac{j-1}{n}\right)+C\left(\frac{i-1}{n}, \frac{j-1}{n}\right) .
$$

Now, we make a simple observation.

Proposition 4.3. Let $\phi_{1, n}, \phi_{2, n}, \ldots, \phi_{n, n} \in L^{1}([0,1])$ be a partition of unity and

$$
k_{n}(x, y)=n \sum_{i=1}^{n} \phi_{i, n}(x) \chi_{i, n}(y),
$$

where $x_{i, n}$ is the characteristic function of interval $[(i-1) / n, i / n]$. Then for every copula $C$,

$$
T_{n}(C)=P_{n} \circ T_{C} \circ P_{n}^{*} .
$$

Proof. It is easy to verify that $k_{n}$ satisfies conditions (3.1) and (3.2). So $P_{n}$ and $P_{n}^{*}$ are Markov operators. Fix $f \in L^{1}([0,1])$, then from (3.3) and (3.4), it follows that

$$
\begin{aligned}
P_{n} \circ T_{C} \circ P_{n}^{*}(f)(x) \\
=\left(P_{n} \circ T_{C}\right)\left(\int_{0}^{1} n \sum_{j=1}^{n} \phi_{j, n}(s) \chi_{j, n}(x) f(s) d s\right) \\
=P_{n}\left(\frac{d}{d x} \int_{0}^{1} C_{, 2}(x, t) n \sum_{j=1}^{n} \chi_{j, n}(t) \int_{0}^{1} \phi_{j, n}(s) f(s) d s d t\right) \\
=\int_{0}^{1} n \sum_{i=1}^{n} \phi_{i, n}(x) \chi_{i, n}(y) \frac{d}{d y} \int_{0}^{1} C_{, 2}(y, t) n \sum_{j=1}^{n} \chi_{j, n}(t) \int_{0}^{1} \phi_{j, n}(s) f(s) d s d t d y \\
=n^{2} \sum_{i, j=1}^{n} \phi_{i, n}(x)\left(\int_{0}^{1} \phi_{j, n}(s) f(s) d s\right) \int_{(i-1) / n}^{i / n} \frac{d}{d y} \int_{(j-1) / n}^{j / n} C_{, 2}(y, t) d t d y \\
=n^{2} \sum_{i, j=1}^{n} \Delta_{i, j}(C) \phi_{i, n}(x) \int_{0}^{1} \phi_{j, n}(s) f(s) d s .
\end{aligned}
$$

This shows that $T_{n}(C)=P_{n} \circ T_{C} \circ P_{n}^{*}$, which completes the proof.

We are interested in the question of when $T_{n}(C) \rightarrow T_{C}$ in the strong operator topology of $L^{1}$ and also we ask when $T_{n}(C) \rightarrow T_{C}$ in the strong operator topology of $L^{p}$ for all $p \in[1, \infty)$. Since $\left\|P_{n}\right\| \leq 1$ and $\left\|P_{n}^{*}\right\| \leq 1$, the next result follows immediately from (4.7). 
COROLLARY 4.4. Let $p \in[1, \infty)$ be given. Suppose that the operators $P_{n}, P_{n}^{*}$ are generated by kernels $k_{n}$ given by (4.6). Assume that $P_{n} \rightarrow I$ and $P_{n}^{*} \rightarrow I$ in the strong operator topology of $L^{p}$. Then $T_{n}(C) \rightarrow T_{C}$ in the strong operator topology of $L^{p}$.

Now, we can formulate sufficient conditions for approximation by copulas corresponding to Markov operators generated by partitions of unity. Using definition (4.6), we can write the assumption (3.5) of Theorem 3.1 as

$$
\lim _{n \rightarrow \infty} n \sum_{i=1}^{n} \iint_{A(\varepsilon)} \phi_{i, n}(x) \chi_{i, n}(y) d x d y=1,
$$

and the assumption (3.6) and (3.7) of Theorem 3.2 as

$$
\begin{aligned}
\lim _{n \rightarrow \infty} n \int_{A(\varepsilon, x)} \phi_{[n y]+1, n}(x) d x=1 & \text { for a.e. } y \in[0,1], \\
\lim _{n \rightarrow \infty} n \sum_{i=1}^{n} \phi_{i, n}(x) \int_{A(\varepsilon, x)} x_{i, n}(y) d y=1 & \text { for a.e. } x \in[0,1],
\end{aligned}
$$

where $[z]$ denotes the largest integer not larger than $z$.

The following corollary is a consequence of Theorem 3.1 and 3.2, Proposition 4.3 and Corollary 4.4 .

COROLLARY 4.5. Let $\phi_{1, n}, \ldots, \phi_{n, n}$ be a partition of unity for $n=1,2, \ldots$ and let $T_{n}(C)$ be a sequence of Markov operators given by (4.4).

(i) If (4.9) holds, then for every copula $C, T_{n}(C) \rightarrow T_{C}$ in the strong operator topology of $L^{1}$.

(ii) If (4.10) and (4.11) hold, then for every copula $C, T_{n}(C) \rightarrow T_{C}$ in the strong operator topology of $L^{p}$ for every $p \in[1, \infty)$.

\section{Applications}

1. A checkerboard Approximation. Let $C$ be a copula and let $n \in N$. Define

$$
\check{C}_{n}(C)(x, y)=n^{2} \sum_{i, j=1}^{n} \Delta_{i, j}(C) \int_{0}^{x} \chi_{i, n}(s) d s \int_{0}^{y} \chi_{j, n}(t) d t .
$$

We call $\check{C}_{n}(C)$ a checkerboard approximation to $C$. The associated Markov operator can be written as

$$
\left(T_{\check{C}_{n}(C)} f\right)(x)=n^{2} \sum_{i, j=1}^{n} \Delta_{i, j}(C) \chi_{i, n}(x) \int_{0}^{1} \chi_{j, n}(y) f(y) d y .
$$

It is easy to see that $\chi_{1, n}, \ldots, \chi_{n, n}$ is a partition of unity for all $n \in N$. If $T_{n}(C)$ is the Markov operator corresponding to this partition of unity, then $T_{n}(C)=T_{\check{C}_{n}(C)}$ holds. An associated sequence of kernels $k_{n}$ is given by

$$
k_{n}(x, y)=n \sum_{i=1}^{n} x_{i, n}(x) x_{i, n}(y) .
$$

We show that the kernels $k_{n}$ satisfy the assumptions of Corollary 4.5. First, we 
check (4.9). Fix $\varepsilon>0$ and $n>1 / \varepsilon$

$$
n \sum_{i=1}^{n} \iint_{A(\varepsilon)} x_{i, n}(x) x_{i, n}(y) d x d y=n \sum_{i=1}^{n} \int_{(i-1) / n}^{i / n} d x \int_{i-1}^{i / n} d y=n \sum_{n=1}^{n} \frac{1}{n^{2}}=1 .
$$

Now, we check (4.10). Fix $y \in[0,1] \backslash Q$. For $n>1 / \varepsilon$, we have $[[n y] / n,([n y]+1) / n] \subset$ $A(\varepsilon, y)$ and

$$
n \int_{A(\varepsilon, y)} x_{[n y]+1, n}(x) d x=n \int_{[n y] / n}^{([n y]+1) / n} d x=1 .
$$

Since kernels $k_{n}$ are symmetrical, (4.11) also holds. Consequently, $T_{\check{C}_{n}(C)} \rightarrow T_{C}$ in the strong operator topology of $L^{p}$ for every $p \in[1, \infty)$.

2. BERNSTEIN APPROXimAtion. We recall that Bernstein polynomials are defined with the help of the following expressions

$$
b_{k, n}(x)=\left(\begin{array}{l}
n-1 \\
k-1
\end{array}\right) x^{k-1}(1-x)^{n-k} \quad \text { for } k=1, \ldots, n .
$$

The polynomials $b_{1, n}, \ldots, b_{n, n}$ form a partition of unity for $n=1,2, \ldots$. We approximate any copula $C$ by

$$
B_{n}(C)(x, y)=n^{2} \sum_{i, j=1}^{n} \Delta_{i, j}(C) \int_{0}^{x} b_{i, n}(s) d s \int_{0}^{y} b_{j, n}(t) d t, \quad n=1,2, \ldots
$$

The associated kernels $k_{n}$ are given by

$$
k_{n}(x, y)=n \sum_{i=1}^{n} b_{i, n}(x) \chi_{i, n}(y)
$$

and the corresponding Markov operator by

$$
T_{B_{n}(C)}(f)(x)=n^{2} \sum_{i, j=1}^{n} \Delta_{i, j}(C) b_{i, n}(x) \int_{0}^{1} b_{j, n}(y) f(y) d y .
$$

We show that the kernels (5.8) satisfy conditions (4.10) and (4.11) of Corollary 4.5. It is easy to verify that

$$
n b_{m, n}(x)=-\left(\sum_{k=1}^{m} b_{k, n+1}(x)\right)^{\prime} \text { for } m=1, \ldots, n .
$$

Hence,

$$
n \int_{0}^{x} b_{m, n}(t) d t=1-\sum_{k=1}^{m} b_{k, n+1}(x) .
$$

It suffices to show (4.10) for small $\varepsilon$. Fix $y \in[0,1] \backslash Q$ and $\varepsilon \in(0, y)$. Using (5.11), we obtain

$$
n \int_{A(\varepsilon, y)} b_{[n y]+1, n}(x) d x=\sum_{k=1}^{[n y]+1} b_{k, n+1}(y-\varepsilon)-\sum_{k=1}^{[n y]+1} b_{k, n+1}(y+\varepsilon) .
$$


Let $\xi_{n}$ be a sequence of independent random variables such that $\operatorname{Prob}\left(\xi_{n}=1\right)=x$ and $\operatorname{Prob}\left(\xi_{n}=0\right)=1-x$ and let $S_{n}=\xi_{1}+\cdots+\xi_{n}$. Then

$$
\sum_{k=1}^{[n y]+1} b_{k, n+1}(x)=\operatorname{Prob}\left(S_{n}<[n y]+1\right)=\operatorname{Prob}\left(\frac{S_{n}}{n}<\frac{[n y]+1}{n}\right) .
$$

From the law of large numbers, it follows that

$$
\lim _{n \rightarrow \infty} \sum_{k=1}^{[n y]+1} b_{k, n+1}(x)= \begin{cases}1, & x<y, \\ 0, & x>y,\end{cases}
$$

and (4.10), now, follows from (5.12) and (5.14). To check (4.11), we need the well-known Bernstein theorem (cf. [1, Ch. 1, Thm. 6.3]).

LEMMA 5.1 (Bernstein). For every continuous function $f:[0,1] \rightarrow R$

$$
\sum_{i=1}^{n} f\left(\frac{i-1}{n-1}\right) b_{i, n}(x) \rightrightarrows f(x)
$$

uniformly on $[0,1]$.

Fix $x \in[0,1]$ and $\varepsilon>0$. Let $J(\varepsilon, x, n)=\{i \in\{1, \ldots, n\}:[(i-1) / n, i / n] \subset A(\varepsilon, x)\}$. We have

$$
n \sum_{i=1}^{n} b_{i, n}(x) \int_{A(\varepsilon, x)} x_{i, n}(y) d y \geq \sum_{i \in J(\varepsilon, x, n)} b_{i, n}(x) .
$$

Let $\eta<\varepsilon, K(\eta, x, n)=\{i \in\{1, \ldots, n\}:(i-1) /(n-1) \in A(\eta, x)\}$, and $f:[0,1] \rightarrow[0,1]$ be a continuous function such that $f(t)=0$ for $t \in[0,1] \backslash A(\eta, x)$ and $f(x)=1$. Using Lemma 5.1, we obtain

$$
\sum_{i=1}^{n} f\left(\frac{i-1}{n-1}\right) b_{i, n}(t) \rightrightarrows f(t)
$$

which gives, for $t=x$,

$$
\sum_{i=1}^{n} f\left(\frac{i-1}{n-1}\right) b_{i, n}(x) \longrightarrow 1
$$

From (4.1), we get

$$
\sum_{i=1}^{n} f\left(\frac{i-1}{n-1}\right) b_{i, n}(x) \leq \sum_{i \in K(\eta, x, n)} b_{i, n}(x) \leq \sum_{i \in J(\epsilon, x, n)} b_{i, n}(x)
$$

for sufficiently large $n$. Combining this with (5.16) and (5.18), we get (4.11). This shows that $T_{B_{n}(C)} \rightarrow T_{C}$ in strong operator topology of $L^{p}$ for every $p \in[1, \infty)$.

3. A tent approximation. We define a sequence of tent functions by

$$
\begin{aligned}
& \phi_{i, n}(x)=\max \left\{0,1-\left|n x-i+\frac{1}{2}\right|\right\} \quad \text { for } i=2, \ldots, n-1, \\
& \phi_{1, n}(x)=\max \left\{0, \min \left\{1, \frac{3}{2}-n x\right\}\right\}, \quad \phi_{n, n}(x)=\phi_{1, n}(1-x) .
\end{aligned}
$$


It is easy to verify that $\phi_{1, n}, \phi_{2, n}, \ldots, \phi_{n, n}$ form a partition of unity for $n=2,3, \ldots$. We approximate a copula $C$ by

$$
D_{n}(C)(x, y)=n^{2} \sum_{i, j=1}^{n} \Delta_{i, j}(C) \int_{0}^{x} \phi_{i, n}(s) d s \int_{0}^{y} \phi_{j, n}(t) d t, \quad n=2,3, \ldots
$$

The associated kernels $k_{n}$ are given by (4.6) and the corresponding Markov operators by

$$
T_{D_{n}(C)}(f)(x)=n^{2} \sum_{i, j=1}^{n} \Delta_{i, j}(C) \phi_{i, n}(x) \int_{0}^{1} \phi_{j, n}(y) f(y) d y .
$$

We show that the kernels (4.6) of this approximation satisfy conditions (4.10) and (4.11) of Corollary 4.5. Fix $y \in[0,1] \backslash Q, \varepsilon \in(0, y)$ and $n>2 / \varepsilon$. Since

$$
\left[\frac{2[n y]-1}{2 n}, \frac{2[n y]+3}{2 n}\right] \subset A(\varepsilon, y) \text {, }
$$

we have

$$
n \int_{A(\varepsilon, y)} \phi_{[n y]+1, n}(x) d x=1,
$$

which gives (4.10). Now, fix $x \in[0,1] \backslash Q, \varepsilon \in(0, x)$ and $n>2 / \varepsilon$. Observe that $\phi_{i, n}(x) \neq$ 0 only for $i_{1}=[n x+(1 / 2)]$ and $i_{2}=[n x+(3 / 2)]$. Also, we have $[(i-1) / n, i / n] \subset$ $A(\varepsilon, x)$ for $i=i_{1}$ and $i=i_{2}$. Hence,

$$
n \sum_{i=1}^{n} \phi_{i, n}(x) \int_{A(\varepsilon, x)} x_{i, n}(y) d y=\phi_{[n x+(1 / 2)], n}(x)+\phi_{[n x+(3 / 2)], n}(x)=1,
$$

which implies (4.11). From Corollary 4.5, it follows that $T_{D_{n}(C)} \rightarrow T_{C}$ in the strong operator topology of $L^{p}$ for every $p \in[1, \infty)$.

\section{REFERENCES}

[1] P. Billingsley, Probability and measure, 2nd ed., Wiley Series in Probability and Mathematical Statistics: Probability and Mathematical Statistics, John Wiley \& Sons, Inc., New York, 1986. MR 87f:60001. Zbl 649.60001.

[2] J. R. Brown, Approximation theorems for Markov operators, Pacific J. Math. 16 (1966), 1323. MR 33\#777.

[3] W. F. Darsow, B. Nguyen, and E. T. Olsen, Copulas and Markov processes, Illinois J. Math. 36 (1992), no. 4, 600-642. MR 94h:60126.

[4] W. F. Darsow and E. T. Olsen, Norms for copulas, Internat. J. Math. Math. Sci. 18 (1995), no. 3, 417-436. MR 96g:46071.

[5] X. Li, P. Mikusiński, H. Sherwood, and M. D. Taylor, On approximation of copulas, Distributions with given marginals and moment problems (V. Benes et al., ed.), Kluwer Acadmic Publishers, 1997, pp. 107-116. Zbl 905.60015.

[6] X. Li, P. Mikusiński, and M. D. Taylor, Strong approximation of copulas, J. Math. Anal. Appl., submitted.

[7] E. T. Olsen, W. F. Darsow, and B. Nguyen, Copulas and Markov operators, Distributions with Fixed Marginals and Related Topics (L. Rueschendrof M. D. Tayler and B. Schweizer, eds.), IMS Lecture Notes and Monograph Series, vol. 28, Institute of Mathematical Sciences, 1996, pp. 244-259. 
[8] A. Sklar, Fonctions de répartition à $n$ dimensions et leurs marges, Publ. Inst. Statist. Univ. Paris 8 (1959), 229-231 (French). MR 23\#A2899.

[9] _ Random variables, joint distribution functions, and copulas, Kybernetika (Prague) 9 (1973), 449-460. MR 499903.

KulPa: DePartment of MATHEmatics, University of Silesia, BANKOWA 14, 40-007 KatowICE, POLAND

E-mail address: tku1pa@ux2.math.us.edu.p1 


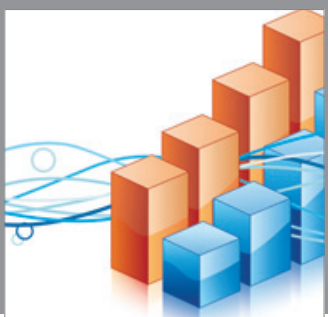

Advances in

Operations Research

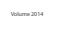

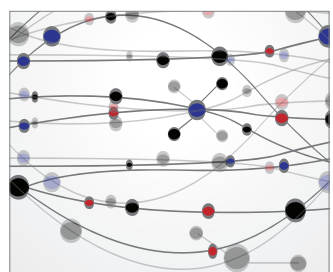

\section{The Scientific} World Journal
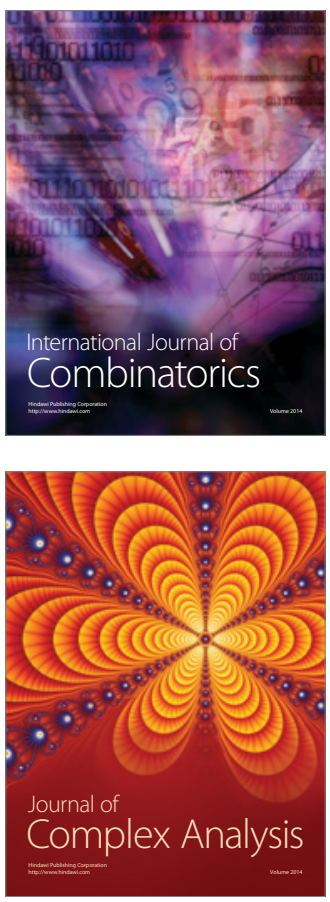

International Journal of

Mathematics and

Mathematical

Sciences
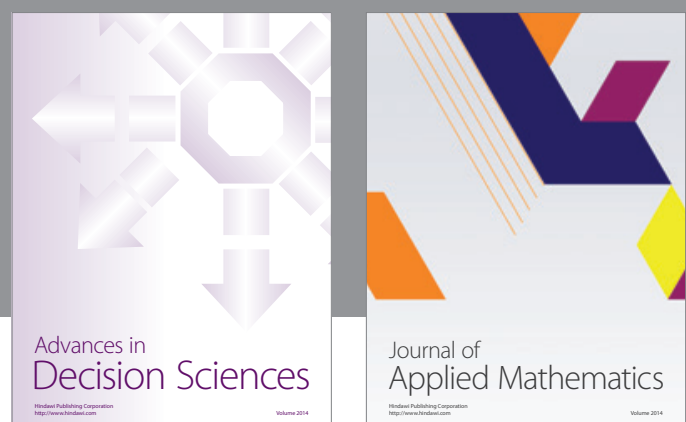

Journal of

Applied Mathematics
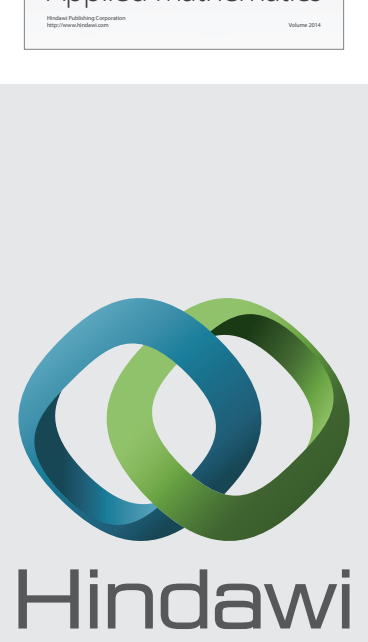

Submit your manuscripts at http://www.hindawi.com
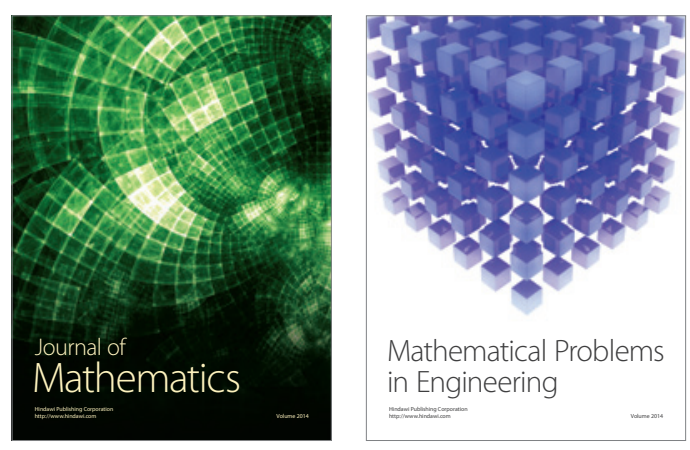

Mathematical Problems in Engineering
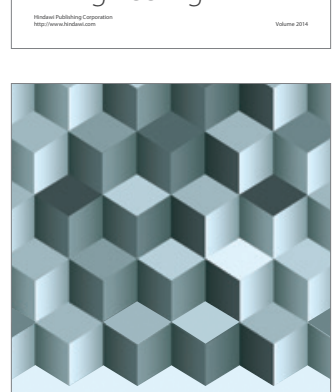

Journal of

Function Spaces
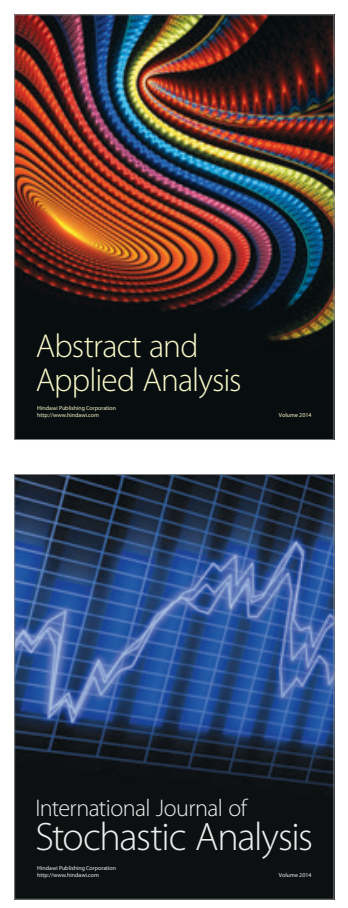

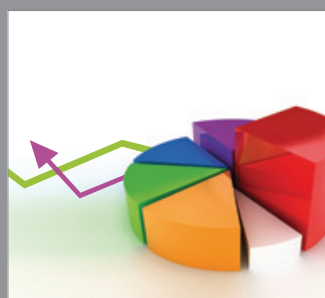

ournal of

Probability and Statistics

Promensencen
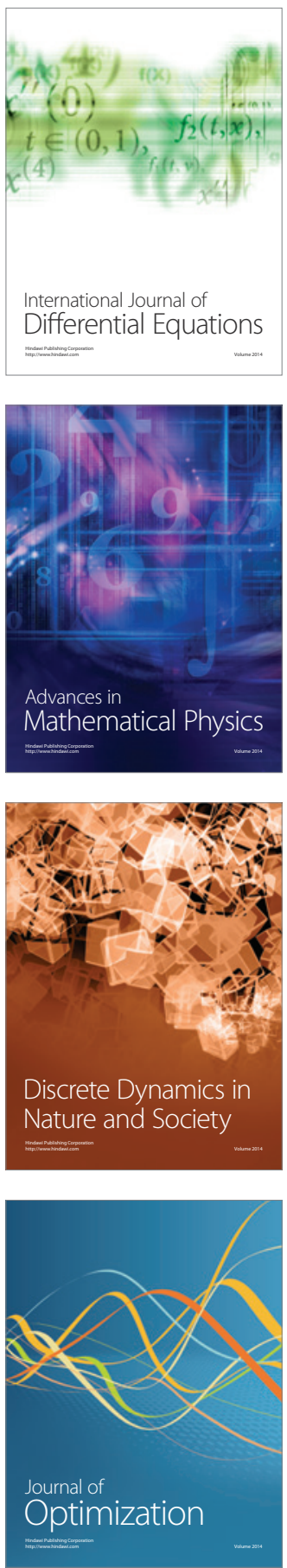